\title{
Efectos de la temperatura, el ambiente lumínico y la escarificación sobre la germinación de semillas de Bromelia serra Griseb. (Bromeliaceae) ${ }^{1}$
}

\begin{abstract}
Graciela Noemí Klekailo ${ }^{2,3 *}$, Daniel Tuesca ${ }^{2,4}$, Ignacio Martín Barberis ${ }^{2,3}$
RESUMEN - Bromelia serra Griseb. (Bromeliaceae) es una hierba terrestre que habita en el sotobosque de bosques abiertos del Chaco y el Cerrado. En este trabajo se analizó el efecto de la temperatura, el clima lumínico y la escarificación sobre la germinación de semillas. Se evaluaron dos regímenes de temperatura $\left(15 / 20{ }^{\circ} \mathrm{C}\right.$ y $20 / 30{ }^{\circ} \mathrm{C}$ ), tres ambientes lumínicos (Luz, Filtro y Oscuridad; $100 \%$ luz y 0.65 R:FR, $100 \%$ luz y 0.09 R:FR y sin luz respectivamente) y dos tratamientos de escarificación (Escarificación durante 1 min. con $\mathrm{H}_{2} \mathrm{SO}_{4}$ al $30 \%$ y Control sin escarificar). La temperatura fue un factor clave ya que solo se registró germinación (una reducida fracción de las semillas y en forma lenta) en el tratamiento a $20 / 30{ }^{\circ} \mathrm{C}$. Las semillas fueron indiferentes a la intensidad de luz, germinando tanto en condiciones de luz como de oscuridad. La calidad de la luz afectó la germinación, registrándose mayores porcentajes de germinación a altas relaciones R:FR. No se detectaron efectos de la escarificación en la germinación de las semillas. Los resultados de este trabajo aportan información básica sobre la germinación de semillas, que resultan útiles para el manejo de la especie.
\end{abstract}

Términos para indexación: calidad de la luz, Chaco, dormición, semillas herbáceas, manejo.

\section{The effects of temperature, light environment and scarification on seed germination of Bromelia serra Griseb. (Bromeliaceae)}

\begin{abstract}
Bromelia serra Griseb. (Bromeliaceae) is a terrestrial herb that grows in the understory of Chaco and Cerrado woodlands. In this study, the effects of temperature, light environment and scarification on seed germination were analyzed. Two temperature regimes $\left(15 / 20^{\circ} \mathrm{C}\right.$ and $\left.20 / 30{ }^{\circ} \mathrm{C}\right)$, three light environments (Light, Filter and Darkness; 100\% light and 0.65 R:FR, 100\% light and $0.09 \mathrm{R}: \mathrm{FR}$, and without light, respectively) and two scarification treatments (Scarification for 1 min. with $\mathrm{H}_{2} \mathrm{SO}_{4}$ at $30 \%$ and Control without scarification). Temperature was a key factor because germination was only recorded (a small fraction of seeds and very slowly) at the $20 / 30^{\circ} \mathrm{C}$ treatment. Seeds were indifferent to light intensity, germinating at full light, as well as at darkness. Light quality affected seed germination, with higher germination percentage at high R:FR. No effect of scarification on seed germination was recorded. The results of this work provide basic information about seed germination that could be useful for the management of this species.
\end{abstract}

Index terms: light quality, Chaco, dormancy, herbaceous seeds, management.

\section{Introducción}

La familia Bromeliaceae comprende tres subfamilias

\footnotetext{
${ }^{1}$ Sometido el 30/03/2012. Aprobado para publicación el 07/08/2012.

${ }^{2}$ Facultad de Ciencias Agrarias, Universidad Nacional de Rosario, Código Postal S2125ZAA, Zavalla, Santa Fe, Argentina.
}

(Pitcairnioideae, Tillandsioideae y Bromelioideae). Dentro de la última subfamilia, el género Bromelia abarca 47 especies principalmente sudamericanas (Smith y Downs, 1979).

\footnotetext{
${ }^{3}$ CONICET (Consejo Nacional de Investigaciones Cinetificas y Técnicas). ${ }^{4}$ CIUNR (Consejo de Investigaciones de la Universidad Nacional de Rosario).

*Autor para correspondencia <gklekailo@gmail.com>
} 
Una de las especies terrestres de más amplia distribución es Bromelia serra Griseb. que se extiende en Argentina, Paraguay, Bolivia y Brasil (Smith y Downs, 1979), en las regiones del Chaco (Zuloaga et al., 2008) y del Cerrado (Forzza et al., 2010). Estas regiones se caracterizan por la presencia de parches leñosos intercalados con áreas graminosas (Lewis, 1991; Cabacinha y de Castro, 2009).

En el Chaco Oriental, B. serra forma densas colonias junto a otras bromeliáceas (Aechmea distichantha) en los parches leñosos de los bosques de Schinopsis balansae (Lewis, 1991; Barberis et al., 2002; Barberis y Lewis, 2005). El sotobosque de estos parches leñosos presenta una elevada heterogeneidad ambiental dada por la micro-topografía y la estructura del canopeo (Barberis et al., 2002), lo cual resulta en condiciones lumínicas y de temperatura muy heterogéneas para el establecimiento, crecimiento y desarrollo de las plantas del sotobosque (Cavallero et al., 2009).

El establecimiento de nuevos individuos depende de las condiciones del hábitat, la disponibilidad de propágulos (vegetativos o reproductivos), así como de los requerimientos particulares de las especies (Harper, 1977; Baskin y Baskin, 1998). En el caso particular de B. serra, las distintas áreas del sotobosque suelen ser colonizadas por individuos producidos vegetativamente mediante estolones. Las plantas adultas alcanzan $0,6 \mathrm{~m}$ de altura y $1-1,5 \mathrm{~m}$ de diámetro, poseen hojas lanceoladas con espinas antrorsas y retrorsas en sus bordes (Smith y Downs, 1979; Barberis et al., 2011). Se observan también individuos generados a partir de semillas, las cuales son de color pardo rojizas, lenticulares y pesan alrededor de $35 \mathrm{mg}$. Sin embargo se desconoce en qué medida las condiciones ambientales (e.g. temperatura, intensidad y calidad lumínica) afectan la capacidad de germinar de las semillas de esta especie.

En los últimos años se han realizado numerosos estudios a campo o en condiciones controladas, sobre germinación de semillas de bromeliáceas. Los mismos abarcan especies de las distintas subfamilias, de distintos hábitos de crecimiento (i.e. epífitas, terrestres, rupícolas) y con distintos medios de dispersión (i.e. viento, animales) (da Rosa y Ferreira, 1998; Pinheiro y Borghetti, 2003; Graham y Andrade, 2004; Mondragón y Calvo-Irabien, 2006; Pompelli, 2006; Vieira et al., 2007; Wiesbauer et al., 2007; Pereira et al., 2009; Anastácio y Santana, 2010; Bonin et al., 2010; Duarte et al., 2010; Dutra et al., 2010; Pereira et al., 2010a,b; Silveira et al., 2010; Vieira y Silveira, 2010; Coelho et al., 2011; Silveira et al., 2011). Varios de estos estudios muestran que las especies de bromeliáceas difieren en los requerimientos de temperatura y luz para germinar (da Rosa y Ferreira, 1998;
Pinheiro y Borghetti, 2003; Graham y Andrade, 2004; Wiesbauer et al., 2007; Pereira et al., 2009; Duarte et al., 2010; Dutra et al., 2010; Pereira et al., 2010b; Silveira et al., 2010; Vieira y Silveira, 2010; Coelho et al., 2011; Silveira et al., 2011).

Los frutos de varias bromeliáceas terrestres son consumidos por numerosas especies de mamíferos que luego dispersan sus semillas (Motta-Junior y Martins, 2002; Beisiegel y Mantovani, 2006). El pasaje de las semillas por el tracto digestivo de estos animales produce un escarificado de la cubierta seminal que podría modificar la capacidad germinativa. Unos pocos estudios han evaluado el efecto de la escarificación sobre la germinación de semillas de bromeliáceas, demostrando la existencia de dormición por presencia de tegumentos impermeables (Pompelli, 2006; Coelho et al., 2011). Los frutos de $B$. serra también son consumidos por varias especies de mamíferos que luego dispersan sus semillas. Se desconoce sin embargo, si sus semillas presentan dormición por presencia de tegumentos impermeables.

Con el objetivo de evaluar la capacidad de Bromelia serra de reclutar individuos a partir de semillas en distintos microclimas generados por la cobertura vegetal heterogénea, se realizaron experimentos en condiciones de laboratorio para determinar los efectos de la temperatura, el ambiente lumínico y la escarificación sobre la germinación de semillas.

\section{Material y Métodos}

Selección de plantas, cosecha de frutos y procesamiento de las semillas: en marzo de 2010 se cosecharon las infrutescencias de 10 individuos reproductivos presentes en parches convexos de un bosque de $S$. balansae ubicado en Las Gamas (21 ${ }^{\circ} 16^{\prime} \mathrm{S}$ y $\left.40^{\circ} 48^{\prime} \mathrm{W}\right)$, provincia de Santa $\mathrm{Fe}$, Argentina. Las infrutescencias se almacenaron en un lugar seco durante una semana hasta su procesamiento. En gabinete, se abrieron los frutos con un bisturí y las semillas fueron removidas, lavadas con agua corriente y luego secadas a temperatura ambiente.

Tratamientos de temperatura, ambiente lumínico $y$ escarificación: se utilizó un diseño factorial con dos tratamientos de temperatura, tres de ambiente lumínico y dos de escarificación. Se colocaron 25 semillas seleccionadas al azar en cajas de Petri de vidrio de $15 \mathrm{~cm}$ de diámetro sobre hojas de papel de filtro húmedo. La germinación de las semillas se realizó en cámaras de crecimiento (Diurnal Growth Chamber, Forma Scientific Inc. Modelo 3740), equipadas con 8 lámparas fluorescentes de $15 \mathrm{~W}$ que generan 
3550 lux/estante y se simuló un fotoperíodo de 16 h. Los tratamientos de temperatura se obtuvieron regulando dos cámaras de crecimiento con dos regímenes de temperaturas $\left(15 / 20{ }^{\circ} \mathrm{C}\right.$ y $20 / 30{ }^{\circ} \mathrm{C}$, respectivamente). Los tratamientos de ambiente lumínico (i.e. Luz, Filtro y Oscuridad) se obtuvieron mediante la manipulación de la cantidad y calidad de luz que recibían las cajas de Petri. En el tratamiento 'Luz' las semillas recibían $100 \%$ de luz sin ningún tipo de filtro. En el tratamiento 'Filtro', las cajas de Petri estaban recubiertas por un filtro (LEE Modelo 124) que permitía obtener una relación de luz roja:roja lejana (i.e. relación R:FR) de 0,09, mientras que el control sin filtro tenía una relación R:FR de 0,65 . Finalmente, en el tratamiento 'Oscuridad' las cajas de Petri fueron colocadas dentro de bolsas plásticas negras. Para los tratamientos de escarificación, la mitad de las semillas fueron sumergidas en $\mathrm{SO}_{4} \mathrm{H}_{2}(30 \%)$ durante un minuto (i.e. tratamiento 'Ácido'), mientras la otra mitad no fue tratada (i.e. tratamiento 'Control'). Esta concentración de $\mathrm{SO}_{4} \mathrm{H}_{2}$ y tiempo de exposición fueron utilizados considerando los resultados obtenidos para Dyckia encholirioides var. encholirioide (Pompelli, 2006).

Se utilizaron cuatro réplicas de 25 semillas en cada combinación de tratamientos. Cada dos días se contaron las semillas germinadas. Las observaciones se realizaron bajo luz verde segura (i.e. green safe light sensu Baskin y Baskin, 1998), y se agregó agua desmineralizada cuando fue necesario. El criterio de germinación fue la protrusión de la radícula. El experimento finalizó a los 80 días, cuando no se registraron nuevas semillas germinadas durante cuatro controles sucesivos

Análisis de los datos: El porcentaje de germinación (G) se calculó como $G=\frac{\text { Ngerm }}{\text { Nsemb }} \times 100$, siendo Ngerm= $\mathrm{N}^{\circ}$ de semillas germinadas, $\mathrm{Nsemb}=\mathrm{N}^{\circ}$ de semillas sembradas. El tiempo medio de germinación (t) se estimó como $t=\frac{\sum n_{i} \times t_{i}}{\sum n_{i}}$, donde $\mathrm{n}_{\mathrm{i}}$ es el número de semillas que germinaron en el tiempo $t_{i} y_{i}$ es el tiempo entre el inicio del experimento y la i-ésima observación. La tasa media de germinación (v) se estimó como la recíproca del tiempo medio de germinación. El coeficiente de variación del tiempo de germinación $(\mathrm{CVt})$ se estimó como el desvío estándar del tiempo de germinación (st) dividido el tiempo medio de germinación multiplicado por 100. Se estimó la incertidumbre del proceso de germinación (U) como $U=-\sum_{i=1}^{k} f_{i} \log _{2} f_{i}$ siendo $f_{i}=\frac{n_{i}}{\sum_{i=1}^{k} n_{i}}$, y la sincronización del proceso de germinación (Z) como $Z=\frac{\sum_{i=1}^{k} C_{n_{i}, 2}}{C_{\sum_{n_{i}, 2}}}$ siendo $C_{n_{i}, 2}=n_{i}\left(n_{i}-1\right) / 2$ (Ranal y Santana, 2006; Ranal et al., 2009). Bajos valores de U indican que la germinación está más concentrada en el tiempo, mientras que los valores de $\mathrm{Z}$ miden el grado de superposición de la germinación (Ranal y Santana, 2006). Finalmente, para expresar los requerimientos de luz para la germinación se utilizó el índice de Germinación Relativa a la Luz (GRL) (Milberg et al., 2000), donde GRL $=\mathrm{GL} /(\mathrm{GO}+\mathrm{GL})$, siendo $\mathrm{GL}=\%$ germinación a la luz, y $\mathrm{GO}=\%$ germinación en oscuridad permanente. Para ello se tomaron los valores promedio de la germinación a la luz y a la oscuridad. El índice de GRL varía entre 0 (germinación sólo en oscuridad) y 1 (germinación sólo a la luz). Si el índice GRL es superior a 0,75 se considera que la especie es dependiente de la luz (fotoblásticas positivas), si es menor a 0,25 se considera repelente de la luz (fotoblásticas negativas), y si el valor está entre 0,25 y 0,75 se considera como indiferente a la luz (Funes et al., 2009).

Para ambos experimentos, el porcentaje de germinación se evaluó con modelos lineales generalizados que consideraban distribución quasi-binomial del error y función logit de ligamiento (Crawley, 2007), mediante el programa R (versión 2.9.0; The R Foundation for Statistical Computing). Las variables $\mathrm{t}, \mathrm{v}, \mathrm{CVt}, \mathrm{U}$ y $\mathrm{Z}$ también se evaluaron con modelos lineales generalizados pero con distribución normal y función 'identidad' de ligamiento (Crawley, 2007). Se compararon modelos estadísticos con y sin el efecto de la interacción entre los factores (i.e. Ambiente Lumínico $\times$ Escarificación) mediante una prueba de F (Crawley, 2007). Dado que el efecto de la interacción no fue significativo ( $p>0,05$ para todas las pruebas), se volvieron a realizar los análisis sólo con los factores principales (Crawley, 2007).

\section{Resultados y Discusión}

Se observó un marcado efecto de la temperatura. En el tratamiento $15 / 20{ }^{\circ} \mathrm{C}$ no se registró germinación inclusive 80 días luego de la siembra (datos no mostrados), mientras que a $20 / 30{ }^{\circ} \mathrm{C}$ las semillas lograron germinar, aunque en un bajo porcentaje (Figura 1). Estos resultados concuerdan con lo registrado para otras especies de la subfamilia Bromelioideae, como Aechmea nudicaulis, Ananas ananassoides, Bromelia antiacantha, Bromelia 
balansae, Bromelia laciniosa, Neoglaziovia variegata, Pseudoananas sagenarius y Streptocalyx floribundus, para las cuales la germinación se vio favorecida entre 25 y $30{ }^{\circ} \mathrm{C}$ (da Rosa y Ferreira, 1998; Pinheiro y Borghetti, 2003; Dutra et al., 2010; Silveira et al., 2010; Vieira y Silveira, 2010; Coelho et al., 2011; Silveira et al., 2011), así como para las subfamilias Pitcairnioideae y Tillandsioideae, en la cuales la germinación estuvo favorecida en el rango de temperaturas comprendido entre 20 y $30{ }^{\circ} \mathrm{C}$, en las especies Pitcairnia albiflos, Dyckia goehringii, Alcantarea imperialis, Pitcairnia flammea, Vriesea heterostachys y
Vriesea penduliflora (Pereira et al., 2009; Duarte et al., 2010; Pereira et al., 2010b). Resultados similares a los obtenidos para Bromelia serra han sido observados para otras especies herbáceas y leñosas del Chaco argentino, donde existe una amplia variación anual de temperatura entre invierno y verano (Funes et al., 2009). Debe tenerse en cuenta que la población de $B$. serra utilizada en este estudio está ubicada al sudeste del Chaco, en el límite sur de distribución de la especie, por lo cual la probabilidad de germinación de semillas y establecimiento de plántulas en invierno es muy baja.
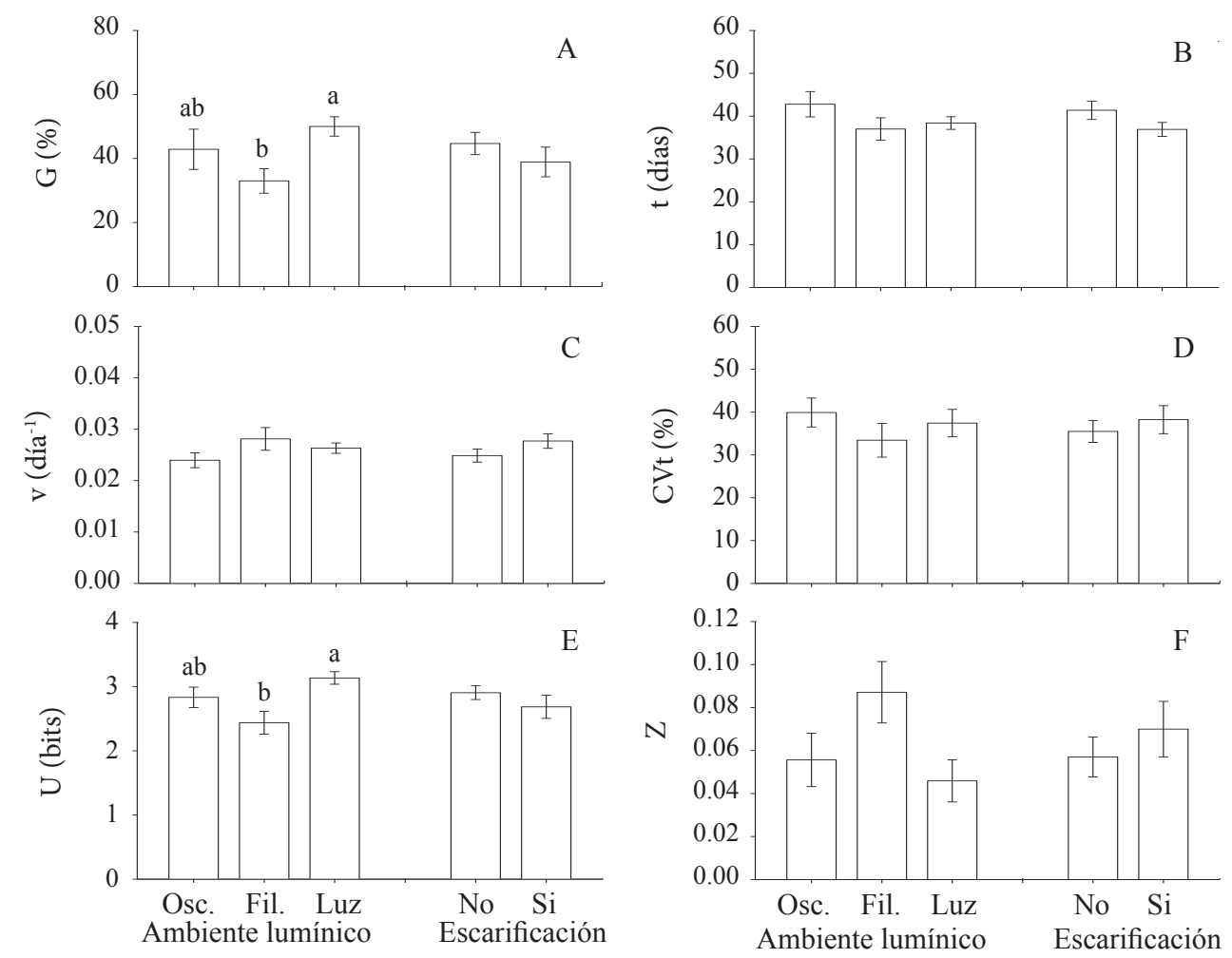

Figura 1. Efectos del ambiente lumínico y la escarificación a temperaturas de $20 / 30^{\circ} \mathrm{C}$ sobre A) el porcentaje de germinación $(\mathrm{G})$, B) el tiempo medio de germinación (t), C) la tasa media de germinación (v), D) el coeficiente de variación en el tiempo de germinación $(\mathrm{CVt}), \mathrm{E})$ la incertidumbre del proceso de germinación $(\mathrm{U})$ y F) el índice de sincronización del proceso de germinación $(Z)$. Letras distintas indican diferencias significativas entre tratamientos (i.e. $p<0,05$ ). Tratamientos de ambiente lumínico: Oscuridad (Intensidad $=0$ lux), Filtro (Intensidad $=3550$ lux y relación R:FR $=0,09)$ y Luz (Intensidad $=3550$ y relación R: FR $=0,65$ ). Tratamientos de escarificación: No (Control) vs. Si (Escarificación con $\mathrm{SO}_{4} \mathrm{H}_{2}(30 \%)$ durante un minuto) y Luz (Intensidad $=3550$ lux y relación R:FR $\left.=0,65\right)$.

*En los gráficos se muestran los promedios \pm el error estándar de la medía.

En el rango de temperatura de $20 / 30{ }^{\circ} \mathrm{C}$ la germinación fue lenta, iniciándose a los 14 días y extendiéndose hasta los 72 días. Smith y Downs (1974) señalan que a $25^{\circ} \mathrm{C}$ la germinación de la mayoría de las bromeliáceas ocurriría entre los 7 y los 28 días.
Sin embargo, estudios posteriores muestran que en la subfamilia Bromelioideae, a la que pertenece $B$. serra, el comportamiento no es uniforme. Por ejemplo, la germinación de Ananas ananassoides también fue lenta (21-27 días) y se extendió por un largo plazo (hasta 
los 86 días) (Anastácio y Santana, 2010), al igual que Bromelia balansae que comenzó a germinar a los 33 días y se prolongó hasta los 110 días (Coelho et al., 2011), mientras que para Nidularium innocentii la germinación se inició a los 10 días (Pereira et al., 2010a). La germinación durante un período prolongado podría ser parte de una estrategia que permitiría a la especie formar bancos de semillas persistentes, sin embargo se desconoce si $B$. serra presenta este comportamiento.

En condiciones de altas temperaturas $\left(20 / 30^{\circ} \mathrm{C}\right)$, se observaron diferencias significativas en el porcentaje de germinación y la incertidumbre del proceso de germinación debido al ambiente lumínico (Tabla 1). Ambas variables fueron mayores en el tratamiento Luz que en el tratamiento Filtro, mientras que en el tratamiento Oscuridad, el comportamiento fue intermedio (Figura 1). Por el contrario, no se observaron diferencias entre tratamientos respecto al tiempo medio de germinación, la tasa media de germinación, el coeficiente de variación en el tiempo de germinación o el índice de sincronización del proceso de germinación (Tabla 1, Figura 1). Al analizar los contrastes entre los distintos tratamientos del Ambiente lumínico, puede apreciarse que no existen diferencias significativas en el porcentaje de germinación de semillas de $B$. serra expuestas a diferentes intensidades de luz (i.e. Luz vs. Oscuridad) (Figura 1). Además el valor del índice de Germinación Relativa a la Luz, que en este estudio fue de 0,54 , sugiere que esta especie es indiferente a la luz. Estos resultados contrastan con lo registrado para otras bromeliáceas, donde las semillas son consideradas fotoblásticas positivas como sucede en Aechmea nudicaulis, Alcantarea imperialis, Ananas ananassoides, Bromelia laciniosa, Dyckia distachya, Pseudoananas sagenarius, Pitcairnia flammea y Streptocalyx floribundus (Pinheiro y Borghetti, 2003; Wiesbauer et al., 2007; Pereira et al., 2009; Dutra et al., 2010; Silveira et al., 2010; Vieira y Silveira, 2010). Sin embargo, también se han registrado especies como Bromelia antiacantha, Tillandsia brachycaulos, $T$. elongata, Vriesea heterostachys y $V$. penduliflora que pueden germinar tanto en la luz como en la oscuridad (da Rosa y Ferreira, 1998; Graham y Andrade, 2004; Pereira et al., 2009). Esto último, coincide con lo observado para la mayoría de las especies herbáceas y leñosas chaqueñas que parecen ser indiferentes a la luz (Funes et al., 2009).

Tabla 1. Resultados de modelos lineales generalizados para evaluar los efectos del ambiente lumínico y la escarificación a temperaturas de $20 / 30{ }^{\circ} \mathrm{C}$ sobre el porcentaje de germinación (G), el tiempo medio de germinación (t), la tasa media de germinación $(\mathrm{v})$, el coeficiente de variación de la germinación $(\mathrm{CVt})$, la incertidumbre del proceso de germinación (U) y el índice de sincronización (Z). Se muestran los grados de libertad (g.l.) del numerador y denominador, la desviación, desviación residual, valores de $\mathrm{F}$ y probabilidad $(\mathrm{P})$ para cada factor. Los valores en negrita muestran valores estadísticamente significativos (i.e. $\mathrm{p}<0,05$ ). Tratamientos de ambiente lumínico: Oscuridad (Intensidad $=0$ lux), Luz (Intensidad $=3550$ lux, y relación R:FR $=0,65)$ y Filtro (Intensidad $=3550$ lux, y relación R:FR = 0,09). Tratamientos de escarificación: No (Control) vs. Si (Escarificación con $\mathrm{SO}_{4} \mathrm{H}_{2}(30 \%)$ durante un minuto).

\begin{tabular}{ccccccc}
\hline \multirow{2}{*}{ Variable } & & \multicolumn{5}{c}{ Desviación } \\
\cline { 5 - 7 } \multirow{2}{*}{$\mathrm{G}$} & Tratamiento & g.l. & Desviación & Residual & $\mathrm{F}$ & $\mathrm{P}$ \\
& Ambiente lumínico & 2,19 & 12,798 & 33,728 & 3,626 & 0,046 \\
& Escarificación & 1,19 & 1,577 & 46,525 & 0,894 & 0,356 \\
\hline \multirow{2}{*}{$\mathrm{t}$} & Ambiente lumínico & 2,19 & 133,935 & 877,32 & 1,635 & 0,221 \\
& Escarificación & 1,19 & 99,097 & 778,22 & 2,419 & 0,136 \\
\hline \multirow{2}{*}{$\mathrm{V}$} & Ambiente lumínico & 2,19 & $6,43 \mathrm{e}-05$ & $4,17 \mathrm{e}-04$ & 1,619 & 0,224 \\
& Escarificación & 1,19 & $3,99 \mathrm{e}-05$ & $3,77 \mathrm{e}-04$ & 2,011 & 0,172 \\
\hline \multirow{2}{*}{$\mathrm{CVt}$} & Ambiente lumínico & 2,19 & 52,661 & 1931,6 & 0,816 & 0,457 \\
& Escarificación & 1,19 & 161,469 & 1879,0 & 0,536 & 0,474 \\
\hline \multirow{2}{*}{$\mathrm{U}$} & Ambiente lumínico & 2,19 & 1,956 & 3,359 & 6,021 & 0,009 \\
& Escarificación & 1,19 & 0,279 & 3,087 & 1,674 & 0,211 \\
\hline \multirow{2}{*}{$\mathrm{Z}$} & Ambiente lumínico & 2,19 & 0,0009 & 0,023 & 3,085 & 0,069 \\
& Escarificación & 1,19 & 0,0074 & 0,022 & 0,819 & 0,377 \\
\hline
\end{tabular}


$\mathrm{Al}$ evaluar el efecto de la calidad de la luz (i.e. Luz vs. Filtro), se encontraron mayores valores de germinación con una relación R:FR alta que con una relación menor (i.e. 0,65 y 0,09 respectivamente) (Figura 1). Estos resultados coinciden con lo registrado por Pereira et al. (2009) para cuatro especies bromeliáceas, donde se encontraron diferencias significativas en los porcentajes de germinación para valores de R:FR similares a los utilizados en este estudio. De manera similar, Graham y Andrade (2004) encontraron que la germinación de las semillas de dos bromeliáceas epífitas fue inhibida por la luz roja lejana. Asimismo, los resultados encontrados en el presente trabajo coinciden con lo expresado por Pons (2000) quien plantea que en muchas especies la exposición de las semillas a la luz durante períodos prolongados tiende a inhibir la germinación cuando la densidad del flujo de fotones (PFD) es alta, especialmente a bajos niveles de relación R:FR.

La incertidumbre del proceso de germinación fue más alta para las semillas de $B$. serra que germinaron en el tratamiento con luz, que para aquellas que germinaron en el tratamiento con filtro, y se observó un comportamiento intermedio en el tratamiento de oscuridad (Tabla 1, Figura 1). Esto indica que tanto en presencia de luz como en oscuridad, la germinación es más heterogénea que en el tratamiento con filtro, sin estar concentrada en un solo momento. Vieira et al. (2007) encontraron que las semillas de Dyckia tuberosa presentaron una alta incertidumbre a bajas temperaturas, tanto en luz como en oscuridad y una baja incertidumbre en la temperatura óptima de germinación $\left(30 / 35^{\circ} \mathrm{C}\right)$. Esto último difiere de los resultados obtenidos en este trabajo, donde la incertidumbre fue alta en la temperatura óptima de germinación. Si como se sugiere, las semillas de $B$. serra permanecen en el banco de semillas del suelo, la alta incertidumbre encontrada (que se traduciría en un período prolongado de germinación) aumentaría las probabilidades de que las plántulas generadas en distintos momentos encuentren condiciones favorables para su desarrollo.

Tampoco se observaron diferencias significativas debidas a la escarificación de las semillas respecto a ninguna de las variables evaluadas (Tabla 1; Figura 1). Estos resultados contrastan con lo observado por Pompelli (2006), quien al exponer semillas de Dyckia encholirioides var. encholirioide a una concentración y tiempo de exposición a $\mathrm{SO}_{4} \mathrm{H}_{2}$ similares a los utilizados en este estudio logró remover la dormición impuesta por las cubiertas seminales. Asimismo, los resultados aquí presentados contrastan con un estudio reciente (Coelho et al., 2011), donde las semillas de Bromelia balansae al ser escarificadas con ácido sulfúrico germinaron en mayor porcentaje. En ese estudio se analizaron los efectos de distintas concentraciones de ácido sulfúrico y distintos tiempos de exposición al mismo. El tiempo de exposición más eficiente para romper la dormición dada por la cubierta seminal fue igual al utilizado en el presente estudio ( 1 minuto). Sin embargo, la concentración de ácido fue más elevada (98\%) que la utilizada en el presente estudio (30\%). Por consiguiente, para corroborar si las semillas de B. serra requieren escarificación deberían realizarse nuevos estudios que evaluaran distintas concentraciones de ácido y tiempos de exposición al mismo.

La información básica sobre los requerimientos de germinación de $B$. serra podría ser de utilidad ya que, de la misma manera que sucede con otras bromeliáceas, las plantas de esta especie podría emplearse para otros fines como por ejemplo, artesanías, industria farmacéutica, usos ornamentales, etc. (Bonin et al., 2010).

\section{Conclusiones}

La temperatura posee un efecto marcado sobre la germinación de semillas de $B$. serra, ya que sólo germinan a 20/30 ${ }^{\circ} \mathrm{C}$. La germinación de semillas no es afectada por la intensidad de la luz, pero sí por la calidad de la misma. La germinación es mayor a altas relaciones R:RL. Finalmente, la escarificación no mejora la germinación, pero dados los resultados observados en otras especies del mismo género sería conveniente realizar nuevos estudios.

\section{Agradecimientos}

Agradecemos a Verónica Albute y Marta Bianchi por su colaboración en la recolección de frutos. Por el apoyo logístico durante la tarea de campo agradecemos la colaboración de los miembros del Centro Experimental Dr. Tito Livio Coppa, Ministerio de la Producción de Santa Fe. Agradecemos al CONICET por el apoyo financiero y a la Facultad de Ciencias Agrarias, Universidad Nacional de Rosario por el uso de las instalaciones.

\section{Referencias}

ANASTÁCIO, M.R.; SANTANA, D.G.D. Germination characteristics of seeds of Ananas ananassoides (Baker) L.B. Sm. (Bromeliaceae). Acta Scientiarum - Biological Sciences, v.32, p.195-200, 2010. http://eduem. uem.br/ojs/index.php/actascibiolsci/article/view/1693 
BARBERIS, I.M.; LEWIS, J.P. Heterogeneity of terrestrial bromeliad colonies and regeneration of Acacia praecox (Fabaceae) in a humidsubtropical-Chaco forest, Argentina. Revista de Biología Tropical, v.53, p.377-385, 2005. http://www.scielo.sa.cr/scielo.php?pid=s0034$77442005000200008 \&$ script $=$ sci_arttext\&tlng=pt

BARBERIS, I.M.; BATISTA, W.B.; PIRE, E.F.; LEWIS, J.P.; LEÓN, R.J.C. Woody population distribution and environmental heterogeneity in a Chaco forest, Argentina. Journal of Vegetation Science, v.13, p.607-614, 2002. http://onlinelibrary.wiley.com/doi/10.1111/j.1654-1103.2002.tb02088.x/abst ract?userisauthenticated $=$ false $\&$ deniedaccesscustomisedmessage $=$

BARBERIS, I.M.; BOCCANELLI, S.; ALZUGARAY, C. Terrestrial bromeliads as seed accumulation microsites in a xerophytic forest of Southern Chaco, Argentina. Bosque, v.32, p.57-63, 2011. http://www.scielo. cl/scielo.php?pid=s0717-92002011000100007\&script=sci_arttext\&tlng=pt

BASKIN, C.C.; BASKIN, J.M. Seeds. Ecology, biogeography, and evolution of dormancy and germination. San Diego: Academic Press, 1998. 666p.

BEISIEGEL, B.M.; MANTOVANI, W. Habitat use, home range and foraging preferences of the coati Nasua nasua in a pluvial tropical Atlantic forest area. Journal of Zoology, v.269, p.77-87, 2006. http:// onlinelibrary.wiley.com/doi/10.1111/j.1469-7998.2006.00083.x/full

BONIN, M.P.; MORAES, C.P.D.; MARTINI, G.D.A.; BENEDITO, P.V.; SOUZA-LEAL, T. Avaliação de tratamentos pré-germinativos com diferentes concentrações de $\mathrm{GA}_{3}$ na germinação de Alcantarea imperialis (Vell.) Harms. Scientia Plena, v.6, p.1-4, 2010. http://www.scientiaplena. org.br/ojs/index.php/sp/article/view/73

CABACINHA, C.D.; CASTRO, S.S. Relationships between floristic diversity and vegetation indices, forest structure and landscape metrics of fragments in Brazilian Cerrado. Forest Ecology and Management, v.257, p.2157-2165. 2009. http://www.sciencedirect.com/science/article/pii/s037811270900142x

CAVALLERO, L.; LÓPEZ, D.; BARBERIS, I.M. Morphological variation of Aechmea distichantha (Bromeliaceae) in a Chaco forest: habitat and sizerelated effects. Plant Biology, v.11, p.379-391. 2009. http://onlinelibrary. wiley.com/doi/10.1111/j.1438-8677.2008.00123.x/full

COELHO, M.D.F.T.B.; VIEIRA, S.N.; CHIG, L.A.; SANTOS, L.W.; ALBUQUERQUE, M.C.D.F.E. Superação da dormência em sementes de Bromelia balansae (Bromeliaceae). Horticultura Brasileira, v.29, p.472-476, 2011. http://www.scielo.br/scielo.php?pid=S010205362011000400005\&script=sci_arttext

CRAWLEY, M.J. The R book. Chichester: John Wiley \& Sons, 2007. 942p.

DA ROSA, S.G.T.; FERREIRA, A.G. Germinação de sementes de espécies medicinais do Rio Grande do Sul: Bromelia antiacantaha Bert., Cuphea carthagenensis (Jacq.) Macbride e Talinum patens (Jacq.) Willdenow. Acta Botanica Brasilica, v.12, p.515-522, 1998. http://www.scielo.br/scielo. php?pid=s0102-33061998000400018\&script=sci_abstract\&tlng=es

DUARTE, E.F.; CARNEIRO, I.F.; SILVA, N.F.D.; GUIMARÃES,
N.N.R. Características físicas e germinação de sementes de Dyckia goehringii Gross \& Rauh (Bromeliaceae) sob diferentes temperaturas. Pesquisa Agropecuaria Tropical, v.40, p.422-429, 2010. http://www. revistas.ufg.br/index.php/pat/article/viewarticle/6037

DUTRA, A.S.; TEÓFILO, E.M.; MEDEIROS FILHO, S. Germinação de sementes de macambira (Bromelia laciniosa Mart. ex Schult). Revista Caatinga, v.23, p.12-17, 2010. http://periodicos.ufersa.edu.br/revistas/ index.php/sistema/article/view/1610

FORZZA, R.C.; COSTA, A.; SIQUEIRA FILHO, J.A.; MARTINELLI, G. Bromeliaceae in lista de espécies da flora do Brasil. Jardim Botânico do Rio de Janeiro. 2010. http://floradobrasil.jbrj.gov.br/2010/fb027621. Acceso en: 20 feb 2012.

FUNES, G.; DÍAZ, S.; VENIER, P. La temperatura como principal determinante de la germinación en especies del Chaco seco de Argentina. Ecología Austral, v.19, p.129-138, 2009. http:/www.scielo.org.ar/scielo. php?script=sci_arttext\&pid=s1667-782X2009000200005

GRAHAM, E.A.; ANDRADE, J.L. Drought tolerance associated with vertical stratification of two co-occurring epiphytic bromeliads in a tropical dry forest. American Journal of Botany, v.91, p.699-706, 2004. http://www.amjbot.org/content/91/5/699.full

HARPER, J.L. Population biology of plants. London: Academic Press, 1977. 892p.

LEWIS, J.P. Three levels of floristical variation in the forests of Chaco. Journal of Vegetation Science, v. 2, p.125-130, 1991. http://onlinelibrary. wiley.com/doi/10.2307/3235905/abstract

MILBERG, P.; ANDERSSON, L.; THOMPSON, K. Large-seeded species are less dependent on light for germination than small-seeded ones. Seed Science Research, v.10, p.99-104, 2000. http://journals. cambridge. org/action/displayabstract? frompage $=$ online $\&$ aid $=693816$

MONDRAGÓN, D.C.; CALVO-IRABIEN, M.L. Seed dispersal and germination of the epiphyte Tillandsia brachycaulos (Bromeliaceae) in a tropical dry forest, Mexico. The Southwestern Naturalist, v.51, p.462-470, 2006. http://www.bioone.org/doi/abs/10.1894/0038-4909(2006)51\%5b4 62:sdagot $\% 5 \mathrm{~d} 2.0 . \operatorname{co} \% 3 \mathrm{~b} 2$

MOTTA-JUNIOR, J.C.; MARTINS, K. The frugivorous diet of the maned wolf, Chrysocyon brachyurus, in Brazil: ecology and conservation. In: LEVEY, D.J.; SILVA, W.R.; GALETTI, M. (Ed). Seed dispersal and frugivory: ecology, evolution and conservation. Campinas: Unicamp, 2002. p.291-303.

PEREIRA, A.R.;ANDRADE,A.C.S.D.; PEREIRA, T.S.; FORZZA, R.C.; RODRIGUES, A.S. Comportamento germinativo de espécies epífitas e rupícolas de Bromeliaceae do Parque Estadual do Ibitipoca, Minas Gerais, Brasil. Revista Brasileira de Botânica, v.32, p.827-838, 2009. http:// www.scielo.br/scielo.php?pid=s0100-84042009000400020\&script $=$ sci arttext\&tlng=pt

PEREIRA, C.; CUQUEL, F.L.; PANOBIANCO, M. Germinação e 
armazenamento de sementes de Nidularium innocentii (Lem.). Revista Brasileira de Sementes, 32, p.36-41, 2010a. http://www.scielo.br/scielo. php?pid=s0101-31222010000200004\&script=sci_arttext\&tlng=es

PEREIRA, A.R.; ANDRADE, A.C.S.; PEREIRA, T.S.; FORZZA, R.C.; RODRIGUES, A.S. Morphological aspects of seed, germination and storage of Pitcairnia albiflos (Bromeliaceae). Seed Science and Technology, v.38, p.79-87, 2010b. http://www.ingentaconnect.com/ content/ista/sst/2010/00000038/00000001/art00008

PINHEIRO, F.; BORGHETTI, F. Light and temperature requirements for germination of seeds of Aechmea nudicaulis (L.) Griesebach and Streptocalyx floribundus (Martius ex Schultes F.) Mez (Bromeliaceae). Acta Botanica Brasilica, v.17, p.27-35, 2003. http://www.scielo.br/ scielo.php?pid=s0102-33062003000100003\&script=sci_arttext

POMPELLI, M.F. Germinação de Dyckia encholirioides var encholirioides (Bromeliaceae, Pitcairnioideae). Floresta e Ambiente, v.13, p.1-9, 2006.

PONS, T.L. Seed responses to light. In: FENNER, M. (Ed). Seeds. The ecology of regeneration in plant communities. Melksham: CAB International, 2000. p.237-260.

RANAL, M.A.; SANTANA, D.G.D. How and why to measure the germination process? Revista Brasileira de Botânica, v.29, p.1-11, 2006. http://www.scielo. br/scielo.php?pid=S0100-84042006000100002\&script=sci_arttext\&tlng=pt

RANAL, M.A.; SANTANA, D.G.; FERREIRA, W.R.; MENDESRODRIGUES, C. Calculating germination measurements and organizing spreadsheets. Revista Brasileira de Botânica, v.32, p.849-855, 2009. http:// www.scielo.br/scielo.php?pid=s0100-84042009000400022\&script $=$ sci_ arttext

SILVEIRA, F.A.D.O.E.; SANTOS, J.C.; FERNANDES, G.W. Seed germination ecophysiology of the wild pineapple, Ananas ananassoides (Baker) L.B.Sm. (Bromeliaceae). Acta Botanica Brasilica, v.24, p.1100-1103, 2010. http://www.scielo.br/scielo. php?pid=S0102-33062010000400026\&script=sci_arttext
SILVEIRA, D.G.; PELACANI, C.R.; ANTUNES, C.G.C.; ROSA, S.S.; SOUZA, F.V.D.; SANTANA, J.R.F.D. Resposta germinativa de sementes de caroá [Neoglaziovia variegata (Arruda) Mez]. Ciência e Agrotecnologia, v.35, p.948-955, 2011. http://www.scielo.br/scielo. php?pid=s1413-70542011000500012\&script=sci_arttext

SMITH, L.B.; DOWNS, R.J. Bromeliaceae, subfamily Pitcairnioideae. In: FLORA NEOTROPICA MONOGRAPH. New York: New York Botanical Garden, 1974. Monog. 14 p. 1-658.

SMITH, L.B.; DOWNS, R.J. Bromeliaceae, subfamily Bromelioideae. In: FLORA NEOTROPICA MONOGRAPH. New York: New York Botanical Garden, 1979. Monog. 14 p.1493-2142.

VIEIRA, B.C.; SILVEIRA, F.A.O. Reproductive phenology, seed germination and ex situ conservation of Pseudananas sagenarius in a semideciduous tropical forest fragment. Plant Species Biology, v.25, p.214-220, 2010. http://onlinelibrary.wiley.com/doi/10.1111/j.1442-1984.2010.00292.x/ abstract?userisauthenticated $=$ false $\&$ deniedaccesscustomisedmessage $=$

VIEIRA, D.C.M.; SOCOLOWSKI, F.; TAKAKI, M. Germinação de sementes de Dyckia tuberosa (Vell.) Beer (Bromeliaceae) sob diferentes temperaturas em luz e escuro. Revista Brasileira de Botânica, v.30, p.183188, 2007. http://www.scielo.br/scielo.php?script=sci_arttext\&pid=s0100$84042007000200003 \&$ lang=pt

WIESBAUER, M.; SCARIOT, E.C.; SASAKI, L.L.; REIS, A. Influência da luz e inundação na germinação de Dyckia distachya Hassler, uma bromélia em vias de extinção. Revista Brasileira de Biociências, v.5, p.717-719, 2007. http://www6.ufrgs.br/seerbio/ojs/index.php/rbb/article/ viewarticle/751

ZuloAgA, F.O.; MORROne, O.; BElgRAnO, M.J. Catálogo de Plantas Vasculares del Cono Sur. Monographs. Missouri: Missouri Botanical Garden, 2008. 3348p. 